(6)

\section{OPEN ACCESS}

Department of Health, Behavior \& Society, Institute for Global Tobacco Control, Johns Hopkins Bloomberg School of Public Health, Baltimore, Maryland, USA

\section{Correspondence to}

Professor Ryan David Kennedy, Department of Health, Behavior \& Society, Institute for Global Tobacco Control, Johns Hopkins Bloomberg School of Public Health, 2213 McElderry Street, 4th Floor, Baltimore, MD 21205 USA; rdkennedy@jhu.edu

Received 18 May 2016 Revised 30 October 2016 Accepted 3 November 2016 Published Online First 30 November 2016

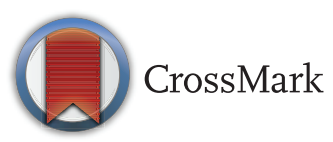

To cite: Kennedy RD Awopegba A, De León $\mathrm{E}$, et al. Tob Control 2017:26:440-445.

\title{
Global approaches to regulating electronic cigarettes
}

\author{
Ryan David Kennedy, Ayodeji Awopegba, Elaine De León, Joanna E Cohen
}

ABSTRACT

Objectives Classify and describe the policy approaches used by countries to regulate e-cigarettes.

Methods National policies regulating e-cigarettes were identified by (1) conducting web searches on Ministry of Health websites, and (2) broad web searches. The mechanisms used to regulate e-cigarettes were classified as new/amended laws, or existing laws. The policy domains identified include restrictions or prohibitions on product: sale, manufacturing, importation, distribution, use, product design including e-liquid ingredients, advertising/promotion/sponsorship, trademarks, and regulation requiring: taxation, health warning labels and child-safety standards. The classification of the policy was reviewed by a country expert.

Results The search identified 68 countries that regulate e-cigarettes: 22 countries regulate e-cigarettes using existing regulations; 25 countries enacted new policies to regulate e-cigarettes; 7 countries made amendments to existing legislation; 14 countries use a combination of new/amended and existing regulation. Common policies include a minimum-age-of-purchase, indoor-use (vape-free public places) bans and marketing restrictions. Few countries are applying a tax to e-cigarettes. Conclusions $A$ range of regulatory approaches are being applied to e-cigarettes globally; many countries regulate e-cigarettes using legislation not written for e-cigarettes.

\section{INTRODUCTION}

Electronic cigarettes, or e-cigarettes, and other electronic nicotine delivery systems (ENDS), alternative nicotine delivery systems (ANDS) or vapourised nicotine products (VNPs) are devices that heat a liquid, commonly propylene glycol and/or glycerol (glycerin), to create an inhalable aerosol that can contain nicotine.

The modern e-cigarette is often credited to Hon Lik, a Chinese pharmacist who invented the product in $2003 ;{ }^{1}$ Lik's design was patented internationally in 2007 (Electronic Atomization Cigarette: US 20070267031 A1). E-cigarettes became commercially available in Europe and the USA around 2006. ${ }^{2}$ Reported use of e-cigarettes has increased dramatically in recent years although globally use remains low; countries that report prevalence of adults using e-cigarettes include $0.3 \%$ in Indonesia, $1.2 \%$ in Italy, $1.9 \%$ in Greece and $5.9 \%$ in Poland. ${ }^{3}$ The value of global sales of ecigarettes was estimated to be 3.5 billion US\$ in $2015,{ }^{4}$ with the largest three markets being the USA, Russia and Germany (collectively representing $60 \%$ of global sales). ${ }^{5}$ E-cigarette products have been marketed by manufacturers as alternative products to combustible tobacco that can be used when cigarettes are not permitted, ${ }^{6}$ or instead of cigarettes. ${ }^{7}$
A number of global tobacco control researchers have stated that e-cigarettes are less harmful than combusted tobacco; ${ }^{8-12}$ however, the impact of e-cigarettes on public health is unclear. This presents specific challenges as jurisdictions determine how to regulate these products.

Studies from around the world suggest that the majority of adults using e-cigarettes are current cigarette users, and some studies suggest that e-cigarette use is often motivated by a desire to quit using combustible cigarettes. ${ }^{12-16}$ However, there is evidence that some non-smokers, including non-smoking youth are also experimenting with e-cigarettes; ${ }^{13}{ }^{17}$ Some public health advocates have raised the concern that e-cigarettes may be a 'starter' or initiation product for youth who will progress to using combustible tobacco products. ${ }^{18}$ It is unclear if non-cigarette smoking youth who report using e-cigarettes would have smoked cigarettes had e-cigarettes not been available, or how long these youth are likely to use e-cigarettes.

Many policy domains can be addressed through e-cigarette regulations. The WHO Tobacco Free Initiative commissioned a report to help countries around the world develop policies to regulate e-cigarettes. ${ }^{19} 20$ This report, published in 2013, provided detailed policy suggestions for countries to regulate e-cigarettes including: banning the use of e-cigarettes anywhere that the use of conventional cigarettes is prohibited, banning the sale of e-cigarettes to anyone who cannot legally buy cigarettes or in any venues where sale of conventional cigarettes is prohibited, apply the same marketing restrictions to e-cigarettes as are applied to conventional cigarettes, ban the practice of cobranding e-cigarette products with cigarettes or marketing in a way that promotes dual use, banning the use of characterising flavours in e-cigarettes, particularly candy and alcohol flavours, banning companies from making claims regarding tobacco-use cessation (until such a time when e-cigarette manufacturers and companies provide sufficient evidence that ENDS products can be used effectively for cessation), and prohibiting e-cigarette companies from making health claims about their products unless approved by appropriate regulatory agencies, and calls for the development of standards for regulating product ingredients and functioning.

In 2014, the WHO Tobacco Free Initiative released a report for the Sixth session of the Conference of the Parties to the WHO Framework Convention on Tobacco Control (FCTC) that included findings from a survey sent to all WHO Member States on ENDS and novel tobacco products. $^{21}$ The survey was completed by 90 countries. When considering ENDS products that contain nicotine, 22 countries reported that ENDS were being classified as tobacco products, 12 countries 
classified ENDS as therapeutic products and 14 countries reported that they are classified as consumer products. This report did not provide country-specific policy approaches. The Conference of the Parties Decision recognised that the parties have used a range of strategies to regulate e-cigarettes including bans on their sale, the adoption of regulation similar to that applicable to the marketing of medicines, their control as tobacco products or no regulation at all. $^{22}$ The WHO report did not address some regulatory issues including nicotine concentrations in products, packaging, including guidance around health warnings.

The authors of the present manuscript sought to identify all country-level policies regulating e-cigarettes and understand how they are being regulated.

\section{METHODS}

This study identified countries using policies to regulate ecigarettes at the national level. Data collection took place between September 2014 and February 2015 and then was regularly updated until October 2016. This involved an iterative method of web-based searching and contacting in-country policy experts, detailed below.

\section{Identifying countries regulating e-cigarettes}

The study team used two general strategies to identify countries regulating e-cigarettes. First, the team searched for policies in a subset of countries including the 90 countries that had participated in the 2014 WHO ENDS and novel tobacco products survey. $^{21}$ An additional 12 high-income countries that are members of the Organisation for Economic Co-operation and Development (OECD) but did not contribute to the WHO survey were also included in the targeted search. The study team theorised that higher income countries might be more likely to have an e-cigarette market. Second, we conducted searches to identify other jurisdictions using policy to regulate e-cigarettes at the country level using Google and Tobacco Watcher (tobaccowatcher.globaltobaccocontrol.org), a surveillance system for tobacco-focused media stories in 23 languages. Search strategies included variant terms for 'electronic cigarettes' and 'regulation' as keywords. These searches yielded media and non-governmental organization (NGO) reports as well as e-cigarette advocacy websites which revealed possible national level e-cigarette regulation. Through this method, the study team identified an additional 21 countries with potential e-cigarette regulation, for a total of 123 countries included in the policy scan.

\section{Identification of e-cigarette regulation documents}

To obtain a copy of each policy, the study team searched the 123 countries' Ministry of Health $(\mathrm{MOH})$ (or similar) websites using variant terms for 'electronic cigarettes' and 'regulation' in English or an official language of the country. Where e-cigarette regulations could not be identified online, the study team contacted in-country tobacco policy experts or $\mathrm{MOH}$ staff to enquire about the existence of policies regulating e-cigarettes in their country. This was carried out using a variety of techniques including sending email requests to the email addresses provided on ministry websites, or sending a note using the 'contact us' web page.

\section{Classification of policy approaches, product classification and domains regulating e-cigarettes}

Copies of the written policies regulating e-cigarettes were reviewed by the study team to understand how e-cigarettes were being regulated. The study team could review policies written in
English, Spanish or French. The team used Google Translate effectively to review regulations in other languages.

First, the study team identified what kind of legal mechanism was being used to regulate e-cigarettes, such as a law, resolution and decree. Second, the team determined if the policy regulating e-cigarettes was a new or amended law that explicitly regulated e-cigarettes, or if e-cigarettes were being regulated using existing legislation. Third, the team reviewed how e-cigarettes were classified through regulation, that is, whether the devices were considered tobacco products, consumer products or medicinal products. Finally, the study team reviewed the content of regulations and identified what regulatory domains were being applied to e-cigarettes including prohibitions or restrictions. The domains were informed by tobacco control regulations and included sale of products including age-of-purchase, advertising/ promotion/sponsorship, distribution, importation, manufacturing, use including vape-free public places, product design including e-liquid ingredients such as flavours and nicotine, taxation, health warning labels (HWLs). We added the categories of, child-safety, and trademarks based on our review of policies.

\section{Verification of regulation}

The study team's country classifications were verified by an in-country expert. In most cases, direct contact through phone or email was made with country bureaucrats (generally with the $\mathrm{MOH}$ ) as well as in-country tobacco policy experts to clarify interpretations of regulation. In many countries, multiple contacts provided guidance and verification.

\section{RESULTS \\ Countries regulating e-cigarettes and their regulatory approaches}

The team identified 68 countries that regulate e-cigarettes at the national level using a range of regulatory mechanisms including laws (new or amended), alerts, circulars, decisions, decrees, notifications, orders, ordinances, rulings and statements. The team identified 22 countries that regulate e-cigarettes using existing regulations; 25 countries that enacted new policies (including laws, rulings, decrees, orders or similar) to regulate e-cigarettes; 7 countries that made amendments to existing legislation and 14 countries that use a combination of new/amended regulations in combination with existing regulation. The identified countries are on six continents and include economies that are classified as high-income, middle-income and low-income. The other 55 countries included in the search did not have national laws regulating e-cigarettes.

The regulatory approaches, product classification and regulatory domains identified for each of the 68 countries are included in table 1 .

\section{Product classification(s)}

In jurisdictions where new or amended policies regulate e-cigarettes, products are generally being classified as 'e-cigarette', or 'electronic cigarette', or 'electronic smoking device' or 'ENDS'. In jurisdictions that are using existing legislation to regulate e-cigarettes, the devices are classified as a range of products including: tobacco, tobacco derivatives, tobacco imitation products, consumer goods, chemical mixtures, drugs, medicinal products or devices and/or poisons/hazardous substances. Countries classify e-cigarettes based on a variety of characteristics including: nicotine content, reported purpose of use, device components and legal language. E-cigarettes are often classified as medical devices if the product's manufacturer makes a claim associated with health. In four countries, nicotine 
Table 1 Global e-cigarette policies: approaches, product classification and regulatory domains addressed (current to October 2016)

\begin{tabular}{|c|c|c|c|}
\hline Country & $\begin{array}{l}\text { Regulatory approach } \\
\text { (es) }\end{array}$ & Product classification(s) & Regulatory domain(s) \\
\hline Argentina & $\begin{array}{l}\text { Decree; existing law; } \\
\text { ruling }\end{array}$ & ENDS, tobacco & Advertising/promotion, distribution, importation, sale, vape-free (implicit) \\
\hline Australia & $\begin{array}{l}\text { Existing law; } \\
\text { statement }\end{array}$ & Poison, consumer good & Advertising/promotion, importation, sale \\
\hline Austria & $\begin{array}{l}\text { Amended law; existing } \\
\text { law; new law }\end{array}$ & $\begin{array}{l}\text { Tobacco-related product, medicinal, } \\
\text { medical device, consumer good }\end{array}$ & $\begin{array}{l}\text { Advertising/promotion/sponsorship, child safety, health warning labelling, } \\
\text { ingredients/flavours, nicotine volume/concentration, reporting/notification, safety/ } \\
\text { hygiene, sale, vape-free }\end{array}$ \\
\hline Bahrain & Existing law; decision & ENDS, tobacco & Advertising/promotion, sale, vape-free \\
\hline Belgium & Existing law & Medicinal, consumer good & Advertising/promotion, distribution, importation, manufacture, sale, vape-free \\
\hline Brazil & Decree; resolution & E-cigarette, tobacco & Advertising/promotion, distribution, importation, sale, vape-free (implicit) \\
\hline $\begin{array}{l}\text { Brunei } \\
\text { Darussalam }\end{array}$ & Existing law & Tobacco (imitation), poison & Importation, sale, vape-free (implicit) \\
\hline Cambodia & Circular & E-cigarette & Importation, sale, vape-free \\
\hline Canada & Existing law; notice & Drug, consumer good & Advertising/promotion, importation, manufacture, sale \\
\hline Chile & Resolution & Medicinal & Unclear \\
\hline Colombia & Existing law & Tobacco (imitation) & Advertising/promotion, manufacture, sale, vape-free \\
\hline Costa Rica & Decree; existing law & ENDS, tobacco (derivative) & Advertising/promotion, minimum age, sale, vape-free \\
\hline Croatia & Existing law & $\begin{array}{l}\text { Consumer good, chemical product, } \\
\text { tobacco (imitation) }\end{array}$ & Advertising/promotion \\
\hline Czech Republic & Existing law & $\begin{array}{l}\text { Medicinal, tobacco (imitation), consumer } \\
\text { good, hazardous substance }\end{array}$ & Advertising/promotion, manufacture, minimum age, sale \\
\hline Denmark & Existing law; new law & E-cigarette, medicinal & $\begin{array}{l}\text { Advertising/promotion/sponsorship, health warning labelling, ingredients/flavours, } \\
\text { minimum age, nicotine volume/concentration, reporting/notification, sale, vape-free }\end{array}$ \\
\hline Ecuador & Decree; existing law & ENDS, tobacco (derivative) & Advertising/promotion, minimum age, sale, vape-free \\
\hline Estonia & New law & Tobacco-related product, E-cigarette & $\begin{array}{l}\text { Advertising/promotion/sponsorship, minimum age, nicotine volume/concentration, } \\
\text { sale }\end{array}$ \\
\hline Fiji & Amended law/decree & E-cigarette & $\begin{array}{l}\text { Advertising/promotion, distribution, importation, manufacture, minimum age, sale, } \\
\text { vape-free }\end{array}$ \\
\hline Finland & New law & $\begin{array}{l}\text { Medicinal, tobacco (imitation/substitute), } \\
\text { tobacco-related product, e-cigarette }\end{array}$ & $\begin{array}{l}\text { Advertising/promotion/sponsorship, child safety, health warning labelling, } \\
\text { importation, ingredients/flavours, minimum age, nicotine volume/concentration, } \\
\text { reporting/notification, safety/hygiene, sale, vape-free }\end{array}$ \\
\hline France & $\begin{array}{l}\text { Amended law; decree; } \\
\text { existing law }\end{array}$ & Medicinal, e-cigarette, consumer good & $\begin{array}{l}\text { Advertising/promotion, child safety, ingredients/flavours, nicotine volume/ } \\
\text { concentration, safety/hygiene, sale }\end{array}$ \\
\hline Germany & New law & $\begin{array}{l}\text { Tobacco-related product, e-cigarette, } \\
\text { consumer good }\end{array}$ & $\begin{array}{l}\text { Advertising/promotion/sponsorship, child safety, health warning labelling, } \\
\text { ingredients/flavours, minimum age, nicotine volume/concentration, reporting/ } \\
\text { notification, safety/hygiene, sale, vape-free }\end{array}$ \\
\hline Greece & Amended law & E-cigarette & Advertising/promotion, distribution, manufacture, sale, vape-free \\
\hline Honduras & Decree; existing law & Tobacco (derivative) & Advertising/promotion, minimum age, vape-free \\
\hline Hungary & Amended law & Consumer good & Advertising/promotion, distribution, importation, sale \\
\hline Iceland & Existing law & Medicinal, consumer product & Advertising/promotion, distribution, manufacture, importation, sale \\
\hline Ireland & Existing law; new law & E-cigarette, medicinal, consumer good & $\begin{array}{l}\text { Advertising/promotion/sponsorship, child safety, health warning labelling, } \\
\text { ingredients/flavours, nicotine volume/concentration, reporting/notification, safety/ } \\
\text { hygiene, sale }\end{array}$ \\
\hline Italy & $\begin{array}{l}\text { Amended law/ } \\
\text { ordinance; decree }\end{array}$ & Tobacco-related product, e-cigarette & $\begin{array}{l}\text { Advertising/promotion/sponsorship, child safety, health warning labelling, } \\
\text { ingredients/flavours, minimum age, nicotine volume/concentration, reporting/ } \\
\text { notification, safety and hygiene, tax }\end{array}$ \\
\hline Jamaica & $\begin{array}{l}\text { Amended law; existing } \\
\text { law }\end{array}$ & ENDS, medicinal & Distribution, importation, manufacture, sale, vape-free \\
\hline Japan & $\begin{array}{l}\text { Existing law; } \\
\text { statement }\end{array}$ & Medicinal & Advertising/promotion, distribution, importation, sale \\
\hline Jordan & Official letter & E-cigarette & Advertising/promotion, importation, manufacture, sale, vape-free \\
\hline Kuwait & Decision & E-cigarette & Distribution, sale \\
\hline Latvia & New law & Tobacco-related product, E-cigarette & $\begin{array}{l}\text { Advertising/promotion/sponsorship, child safety, health warning labelling, } \\
\text { ingredients/flavours, nicotine volume/concentration, reporting/notification, safety/ } \\
\text { hygiene, sale, tax }\end{array}$ \\
\hline Lebanon & Decision & E-cigarette & Distribution, importation, sale \\
\hline Lithuania & New law & Tobacco-related products, e-cigarette & $\begin{array}{l}\text { Minimum age, advertising/promotion/sponsorship, child safety, health warning } \\
\text { labelling, ingredients/flavours, nicotine volume/concentration, reporting/notification, } \\
\text { safety/hygiene, sale, vape-free }\end{array}$ \\
\hline Malaysia & Existing law & Poison, electrical appliance & Distribution, importation, minimum age, sale \\
\hline Malta & Legal notice & Tobacco-related product, e-cigarette & $\begin{array}{l}\text { Advertising/promotion/sponsorship, child safety, health warning labelling, } \\
\text { ingredients/flavours, nicotine volume/concentration, reporting/notification, safety/ } \\
\text { hygiene, sale, vape-free }\end{array}$ \\
\hline
\end{tabular}


Table 1 Continued

\begin{tabular}{|c|c|c|c|}
\hline Country & $\begin{array}{l}\text { Regulatory approach } \\
\text { (es) }\end{array}$ & Product classification(s) & Regulatory domain(s) \\
\hline Mauritius & Existing law & Tobacco (imitation) & Distribution, sale \\
\hline Mexico & Existing law & Tobacco (imitation) & Advertising/promotion, distribution, manufacture, sale \\
\hline Nepal & Notification & ENDS & Advertising/promotion, vape-free \\
\hline Netherlands & $\begin{array}{l}\text { Existing law; decree; } \\
\text { order }\end{array}$ & $\begin{array}{l}\text { E-cigarette, medicine, tobacco-related } \\
\text { product, consumer good }\end{array}$ & $\begin{array}{l}\text { Advertising/promotion/sponsorship, child-safety, health warning labelling, } \\
\text { ingredients/flavours, minimum age, nicotine volume/concentration, reporting/ } \\
\text { notification, safety and hygiene, sale }\end{array}$ \\
\hline New Zealand & Existing law & Medicinal, tobacco, consumer good & Advertising/promotion, distribution, importation, sale \\
\hline Nicaragua & Existing law & Tobacco (imitation) & Importation, manufacture, sale \\
\hline Norway & Existing law & Medicinal, tobacco surrogate, e-cigarette & Advertising/promotion, importation, minimum age, sale \\
\hline Oman & Decision & E-cigarette & Distribution, sale \\
\hline Panama & Decree & ENDS, tobacco (imitation) & Advertising/promotion, distribution, importation, sale, vape-free \\
\hline Philippines & Order & Medicinal, medical device & Child-safety, sale, vape-free \\
\hline Poland & New law & Chemical mixture, Consumer good & Unclear \\
\hline Portugal & Existing law & E-cigarette & $\begin{array}{l}\text { Advertising/promotion/sponsorship, child safety, health warning labelling, } \\
\text { ingredients/flavours, minimum age, nicotine volume/concentration, reporting/ } \\
\text { notification, safety/hygiene, sale, tax, vape-free }\end{array}$ \\
\hline Qatar & Circular; decision & E-cigarette & Advertising/promotion, distribution, importation, sale \\
\hline $\begin{array}{l}\text { Republic of } \\
\text { Korea }\end{array}$ & $\begin{array}{l}\text { Amended Law; } \\
\text { existing law }\end{array}$ & Tobacco, consumer good & Advertising/promotion, health warning labelling, minimum age, tax, vape-free \\
\hline Saudi Arabia & Decision & E-cigarette & Advertising/promotion, sale \\
\hline Seychelles & Existing law & Tobacco (imitation) & Advertising/promotion, distribution, importation, manufacture, sale \\
\hline Singapore & Existing law & Tobacco (imitation) & Distribution, importation, sale \\
\hline Slovakia & Amended law & $\begin{array}{l}\text { Tobacco/tobacco-free product intended to } \\
\text { smoked }\end{array}$ & Advertising/promotion, minimum age, vape-free \\
\hline South Africa & Ruling & Medicinal & Sale \\
\hline Spain & Amended law & ENDS & Advertising/promotion, minimum age, vape-free \\
\hline Suriname & New law & ENDS & Distribution, importation, sale \\
\hline Switzerland & $\begin{array}{l}\text { Existing law; } \\
\text { statement }\end{array}$ & E-cigarette, consumer good & Importation, sale \\
\hline Thailand & $\begin{array}{l}\text { Existing law; } \\
\text { notification; order }\end{array}$ & $\begin{array}{l}\text { 'Modern' medicinal, tobacco (imitation), } \\
\text { e-cigarette }\end{array}$ & Importation, manufacture, sale \\
\hline Togo & New law & Tobacco (derivative) & Advertising/promotion, minimum age, tax, vape-free \\
\hline Turkey & $\begin{array}{l}\text { Amended law; circular; } \\
\text { notice }\end{array}$ & Tobacco, e-cigarette & Sale, advertising/promotion, importation, vape-free \\
\hline Ukraine & Amended Law & E-cigarette & Sale, vape-free \\
\hline $\begin{array}{l}\text { United Arab } \\
\text { Emirates }\end{array}$ & Decision & E-cigarette & Advertising/promotion, importation, sale \\
\hline UK & $\begin{array}{l}\text { Existing law; new law; } \\
\text { statement }\end{array}$ & E-cigarette, medicinal, consumer good & $\begin{array}{l}\text { Advertising/promotion/sponsorship, child safety, health warning labelling, } \\
\text { ingredients/flavours, minimum age, nicotine volume/concentration, reporting/ } \\
\text { notification, safety and hygiene, tax }\end{array}$ \\
\hline USA & New law & Tobacco product & $\begin{array}{l}\text { Advertising/promotion, child-safety, health warning labelling, minimum age, } \\
\text { reporting/notification }\end{array}$ \\
\hline Uruguay & Amendment/decree & Electronic smoking device & Advertising/promotion, importation, sale, trademarks \\
\hline Venezuela & Alert, existing law & $\begin{array}{l}\text { Medicinal, tobacco (derivative), consumer } \\
\text { good }\end{array}$ & Advertising/promotion, sale, vape-free \\
\hline Vietnam & Existing law & Tobacco & Advertising/promotion, minimum age, vape-free \\
\hline
\end{tabular}

ENDS, electronic nicotine delivery systems.

is classified as a controlled poison or hazardous substances (Australia, Brunei Darussalam Czech Republic and Malaysia). Brunei Darussalam classifies nicotine liquid as a poison if the nicotine concentration is above $7.5 \%$.

In 37 countries, e-cigarettes could meet multiple product classifications. For example, in Thailand, these products could be classified as e-cigarettes, medicinal products or products that imitate tobacco depending on which regulation is applied.

\section{E-cigarette regulation policy domain(s)}

The study team identified the following domains of regulation including product prohibitions or restrictions related to e- cigarette: manufacturing, distribution, importation, sale including where sales are allowed and minimum age of purchase, use restrictions including vape-free public places, advertising, promotion, and sponsorship, taxation, trademark, health warning labelling, ingredients/flavors, safety/hygiene, reporting/ notification, nicotine volume/concentration and child-safety packaging (table 1).

The sale of e-cigarettes is banned in 25 countries; market authorisation is required in 17 other countries. In countries permitting the sale of e-cigarettes, minimum age-of-purchase policies are common. There are 23 countries with policies that are consistent with the age of purchase for combustible cigarettes 
which is 18 years in; 19 years in the Republic of Korea and 21 years in Honduras.

E-cigarette use is banned in enclosed public spaces such as bars, restaurants and other workplaces in 25 countries. Advertising and promotion of e-cigarettes are banned in 35 countries; some countries did not have explicit advertising and promotion bans, but contend that such bans are inherent within their bans on sale, including Argentina and Australia.

We identified 14 countries that require e-cigarettes to have a HWL, and 13 that regulate ingredients and flavours that can be used in e-cigarettes. Nicotine concentrations cannot exceed $20 \mathrm{mg} / \mathrm{mL}$ of e-liquid in 14 countries. Child-safety standards for e-cigarettes and/or e-liquid bottles are required in 11 countries. Manufacturing standards for e-cigarettes marketed as medicines are required in 13 countries. Regulations on importation of e-cigarettes are in place in 14 countries. The distribution of e-cigarettes marketed as medicines is regulated in 21 countries. Italy, Latvia, Portugal, Republic of Korea, Togo and the UK apply a tax to e-cigarettes. Manufacturers/retailers are required to notify the appropriate authority prior to marketing e-cigarettes in 13 countries. Certain safety standards (eg, using materials/chemicals of high quality) are required for e-cigarettes or e-liquids in 26 countries. Venezuela prohibits the registration of e-cigarettes as a brand/patent.

\section{DISCUSSION}

Our scan identified 68 countries that regulate e-cigarettes through a variety of legal mechanisms. Generally, countries regulate e-cigarettes based on their classification as tobacco, medicinal or consumer products. Some countries are using more than one classification for e-cigarettes resulting in multiple regulatory approaches for these products. Policies that were common included banning the sale of e-cigarettes, and expanding tobacco control laws to e-cigarettes including vape-free public places and age of purchase laws that mirror laws for cigarettes. Of the countries identified regulating e-cigarettes, about a third do not have regulations specifically written for ecigarettes, rather they apply existing tobacco control regulations to these products. This may or may not be consistent with the intent of the original laws.

Some policies that have been adopted for tobacco products, such as HWLs, present interesting challenges for e-cigarettes given that there is a wide variety of devices and packaging. Further, there is little understanding about what health warnings should be included on e-cigarettes. We identified very few examples of policies that are regulating e-cigarette sponsorship outside the European Union. Very few countries are applying taxes to e-cigarettes or the liquids. There were no policies regulating e-liquid ingredients beyond nicotine. Some countries, all in Europe, have introduced policies to address flavourings; this is important given that there is evidence that some chemicals used to flavour e-liquids, including diacetyl, are associated with lung disease. ${ }^{23}$

This study did not review government websites for every country in the world; however, based on the findings from our general search strategy it appears that most of the countries in the world are not regulating e-cigarettes using national regulatory approaches.

In May 2016, the European Union's Tobacco Products Directive addressed the regulation of e-cigarettes across the 28 member states. This directive includes a range of policy domains, including that nicotine-containing liquid can only be sold if the nicotine concentration does not exceed $20 \mathrm{mg} / \mathrm{mL}^{24}$ Countries in the EU are at different stages of policy implementation and in some jurisdictions aspects of the directive are being challenged.

This study has several limitations including the fact that some national policies may not have been identified using our search strategy. Further, policies to regulate e-cigarettes may be implemented at a subnational level including clean air laws (vape-free) and age-of-purchase requirements, so this specific policy scan does not fully represent the scale or scope of e-cigarette regulation around the world. Although we confirmed countries' policies with government bureaucrats in the respective country, there could still be misinterpretations about how the policies are being applied. Further, this policy scan focuses on the policies that are 'on the books'; it is important to note that there might not be full implementation and/or compliance with these policies 'on the ground'.

In tobacco control, and other areas of public health, we have seen the benefit of learning from natural experiments comparing jurisdictions with different policy environments. This policy scan may support the identification of jurisdictions to further study and understand how policies impact e-cigarette use, tobacco use and public health.

The evidence on the harms and benefits of e-cigarettes has not yet been clearly established. As the evidence base grows, we expect additional activity in the regulatory arena.

The state of global e-cigarette regulation will be updated and reported at globaltobaccocontrol.org/e-cigarette/country-lawsregulating-e-cigarettes.

\section{What this paper adds}

- This study scanned the globe to identify which countries are regulating e-cigarettes, what regulatory mechanisms are being used and what regulatory domains are being applied.

- We identified 68 countries regulating e-cigarettes-the most common forms of regulation included sale bans, use restrictions (vape-free public places), age-of-purchase requirements and advertising and promotion bans.

- Few countries are applying a tax to e-cigarettes.

- Many countries are regulating e-cigarette products with legislation that was written before e-cigarettes were on the market.

Acknowledgements The authors thank the $100+$ government staff who helped by identifying the laws or other policies being used to regulate e-cigarettes in their country and confirming our classification.

Contributors JEC and RDK conceived this paper. Data collection was conducted by $A A$ and EDL; organisation of data was conducted by AA, EDL and RDK. RDK authored the paper with major contributions from all authors. All authors approved the final draft.

Funding The funding was provided by Robert Wood Johnson Foundation, Grant Numbers: 72208 and 72390, with some personnel supported through a grant from the Bloomberg Initiative to Reduce Tobacco Use.

Competing interests None declared.

Provenance and peer review Not commissioned; externally peer reviewed.

Data sharing statement The state of global e-cigarette regulation will be updated and reported at: globaltobaccocontrol.org/e-cigarette/country-lawsregulating-e-cigarettes.

Open Access This is an Open Access article distributed in accordance with the Creative Commons Attribution Non Commercial (CC BY-NC 4.0) license, which permits others to distribute, remix, adapt, build upon this work non-commercially, and license their derivative works on different terms, provided the original work is properly cited and the use is non-commercial. See: http://creativecommons.org/ licenses/by-nc/4.0/ 


\section{REFERENCES}

1 Kaisar MA, Prasad S, Liles T, et al. A decade of e-cigarettes: limited research \& unresolved safety concerns. Toxicology 2016;365:67-75.

2 Rahman MA, Hann N, Wilson A, et al. Electronic cigarettes: patterns of use, health effects, use in smoking cessation and regulatory issues. Tob Induc Dis 2014;12:21.

3 Breland A, Soule E, Lopez A, et al. Electronic cigarettes: what are they and what do they do? Ann N Y Acad Sci 2017;1394:5-30.

4 Mincer J. E-cigarette usage surges in past year: Reuters/Ipsos Poll. Reuters, 2015 http://www.reuters.com/article/us-usa-ecigarette-poll-analysisidUSKBN00Q0CA20150610 (accessed 13 Oct 2016).

5 MacGuill S. Euromonitor International 'E-cigarettes'. http://blog.euromonitor.com/ 2012/11/e-cigarettes-a-us2-billion-global-industry-who-should-be-worried.html (accessed 14 Oct 2016)

6 Stanford School of Medicine. Stanford Research into the Impact of Tobacco Advertising-Smoke Anywhere Stanford Tobacco Research. http://tobacco.stanford.


ecigs_img20834.php\&theme_file=fm_ecigs_mt025.php\&theme_name= Freedom\&subtheme_name $=$ Smoke $\% 20$ Anywhere $\% 2$ Stanford $\% 20$ Tobacco $\%$ 20Research (accessed 13 Oct 2016).

7 Stanford School of Medicine. Stanford Research into the Impact of Tobacco Advertising —Switch. http://tobacco.stanford.edu/tobacco_main/images_ecigs.php?

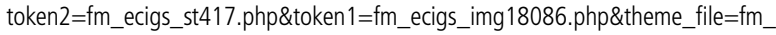
ecigs_mt043.php\&theme_name=Helps $\% 20$ You\%20Quit\&subtheme_name=Switch (accessed 13 Oct 2016).

8 Levy DT, Cummings KM, Villanti AC, et al. A framework for evaluating the public health impact of e-cigarettes and other vaporized nicotine products. Addiction 2017;112:8-17.

9 Knight-West 0 , Bullen C. E-cigarettes for the management of nicotine addiction. Subst Abuse Rehabil 2016;7:111-18.

10 Nutt DJ, Phillips LD, Balfour D, et al. E-cigarettes are less harmful than smoking. Lancet 2016;387:1160-2.

11 Farsalinos KE, Poulas K, Voudris V, et al. Electronic cigarette use in the European Union: analysis of a representative sample of 27460 Europeans from 28 countries. Addiction 2016:111:2032-40.

12 Dockrell M, Morison R, Bauld L, et al. E-cigarettes: prevalence and attitudes in Great Britain. Nicotine Tob Res 2013;15:1737-44.
13 Czoli CD, Hammond D, White CM. Electronic cigarettes in Canada: prevalence of use and perceptions among youth and young adults. Can J Public Health 2014; 105:e97-102.

14 Li J, Newcombe R, Walton D. The prevalence, correlates and reasons for using electronic cigarettes among New Zealand adults. Addict Behav 2015;45:245-51.

15 Vardavas Cl, Filippidis FT, Agaku IT. Determinants and prevalence of e-cigarette use throughout the European Union: a secondary analysis of 26,566 youth and adults from 27 countries. Tob Control 2015;24:442-8.

16 Delnevo CD, Giovenco DP, Steinberg MB, et al. Patterns of electronic cigarette use among adults in the United States. Nicotine Tob Res 2016;18:715-19.

17 Gallus S, Lugo A, Pacifici R, et al. E-cigarette awareness, use, and harm perceptions in Italy: a National Representative Survey. Nicotine Tob Res 2014;16:1541-8.

18 Campaign for Tobacco Free Kids, Press Release: CDC survey finds youth e-cigarette use more than doubled from 2011-2012, shows urgent need for FDA Regulation. http://www.tobaccofreekids.org/press_releases/post/2013_09_05_ecigarettes (accessed 13 Oct 2016)

19 Grana R, Benowitz N, Glantz SA. Background paper on e-cigarettes (electronic nicotine delivery systems). World Health Organization report. Geneva: World Health Organization, 2013. http://escholarship.org/uc/item/13p2b72n (accessed 6 Aug 2014) (archived at http://www.webcitation.org/6SmN0NLgW and http://www. webcitation.org/6SmNONLgp on 22 September 2014).

20 Grana R, Benowitz N, Glantz SA. E-cigarettes: a scientific review. Circulation 2014:129:1972-86.

21 World Health Organization. Electronic nicotine delivery systems. Sixth session, Moscow, Russian Federation, 13-18 October 2014, Provisional Agenda Item 4.4.2. http://apps.who.int/gb/fctc/PDF/cop6/FCTC_COP6_10-en.pdf?ua=1 (accessed 13 Oct 2016).

22 World Health Organization Framework Convention on Tobacco Control, DECISION. Fifth plenary meeting, 18 October 2014. apps.who.int/gb/fctc/PDF/cop6/FCTC COP6(9)-en.pdf (accessed 30 Oct 2016)

23 Allen JG, Flanigan SS, LeBlanc $M$, et al. Flavoring chemicals in e-cigarettes: diacetyl, 2,3-pentanedione, and acetoin in a sample of 51 products, including fruit-, candy-, and cocktail-flavored e-cigarettes. Environ Health Perspect 2016;124:733-9.

24 European Union. Directive 2014/40/EU of the European Parliament and the Council. 3 April 2014. http://ec.europa.eu/health/tobacco/docs/dir_201440_en.pdf (accessed 13 Oct 2016) 